\title{
Electron-vibration coupling constants in positively charged fullerene.
}

\author{
Nicola Manini ${ }^{1,2 *}$, Andrea Dal Corso ${ }^{1,2}{ }^{\prime}$ \\ Michele Fabrizio ${ }^{1,2,3 \ddagger}$ and Erio Tosatti ${ }^{1,2,3 \S}$ \\ ${ }^{1}$ Istituto Nazionale di Fisica della Materia (INFM), \\ Unità Trieste SISSA, \\ 2 International School for Advanced Studies (SISSA), \\ Via Beirut 4, I-34013 Trieste, Italy \\ 3 International Centre for Theoretical Physics (ICTP), \\ P.O. Box 586, I-34014 Trieste, Italy
}

April 10, 2001

\begin{abstract}
Recent experiments have shown that $\mathrm{C}_{60}$ can be positively field-doped. In that state, fullerene exhibits a higher resistivity and a higher superconducting temperature than the corresponding negatively doped state. A strong intramolecular hole-phonon coupling, connected with the Jahn-Teller effect of the isolated positive ion, is expected to be important for both properties, but the actual coupling strengths are so far unknown. Based on density functional calculations, we determine the linear couplings of the two $a_{g}$, six $g_{g}$, and eight $h_{g}$ vibrational modes to the $H_{u}$ HOMO level of the $\mathrm{C}_{60}$ molecule. The couplings predict a $D_{5}$ distortion, and an $H_{u}$ vibronic ground state for $\mathrm{C}_{60}^{+}$. They are also used to generate the dimensionless coupling constant $\lambda$ which controls the superconductivity and the phonon contribution to the electrical resistivity in the crystalline phase. We find that $\lambda$ is 1.4 times larger in positively-charged $\mathrm{C}_{60}$ than in the negatively-doped case. These results are discussed in the context of the available transport data and superconducting temperatures. The role of higher orbital degeneracy in superconductivity is also addressed.
\end{abstract}

\footnotetext{
*E-mail: nicola.manini@mi.infm.it

${ }^{\dagger}$ E-mail: dalcorso@sissa.it

${ }^{\ddagger}$ E-mail: fabrizio@sissa.it

$\S$ E-mail: tosatti@sissa.it
} 


\section{Introduction}

Recent breakthrough experiments (Schön et al. 2000) have shown that a monolayer of $\mathrm{C}_{60}$ can be positively field-doped, a goal hardly realized chemically so far (Datars and Ummat 1995). In that state, fullerene exhibits a higher resistivity than for negative doping, and becomes superconducting with critical temperatures that can exceed $50 \mathrm{~K}$, about a factor 5 higher than the corresponding negative field-doped state. The general belief is that superconductivity in the fullerenes is related to a strong intra-molecular electron-phonon coupling, connected with the Jahn-Teller effect of the isolated ion (Antropov et al. 1993). Unlike the negative doping case, where both calculations (Antropov et al. 1993, Varma et al. 1991, Lannoo et al. 1991) and fits to data (Gunnarsson et al. 1995) exist, no quantitative evaluation of the actual Jahn-Teller coupling strengths is so far available for the positive fullerene ions.

In this work we undertake the task of determining the electron-vibration linear couplings for the $H_{u} \mathrm{HOMO}$ level of the $\mathrm{C}_{60}$ molecule, along with that of spelling out some of the consequences for resistivity and for superconductivity. For that purpose we use density functional electronic structure calculations, yielding accurate molecular vibration frequencies and eigenvectors for the two $a_{g}$, six $g_{g}$, and eight $h_{g}$ modes that couple linearly to the electronic $H_{u}$ state. Knowing the form of the JahnTeller coupling matrices, we distort the molecule and extract the coupling constants from the calculated level shifts and splittings. As a parallel check, we repeat a similar calculation for the negative, electron doping case, where the $a_{g}$ and $h_{g}$ modes couple linearly to the $T_{1 u}$ LUMO level of the $\mathrm{C}_{60}$ molecule. The couplings obtained for negatively doped $\mathrm{C}_{60}$ are rather similar to those that can be found in the literature, and just represent a fresher, state-of-the-art theoretical determination. The holevibration couplings of positively doped $\mathrm{C}_{60}$ are new, and can be put to use in a variety of manners, including predicting or explaining properties of molecular ions, such as photoemission (Brühwiler et al. 1997) and IR/Raman spectra. That is a task that we propose to consider in the near future.

The couplings obtained can also be used to determine the dimensionless electronphonon coupling constant $\lambda$ relevant for the superconductivity as well as for the vibron contribution to the high temperature $T$-linear resistivity in the crystalline phase. Comparing values for positive and negative doping we find that for positive doping $\lambda$ is a factor 1.4 larger than for negative doping. These results provide a starting point for a discussion and comparison with the experimental findings.

This paper is organized as follows: the notation is set up in Sect. 2; the ab-initio calculation and results for the molecular ion are described is Sect. 3; in Sect. 16 we sketch the calculation of the resistivity in a band-degenerate case; Sect. 5 presents a formulation for superconductivity in that case; Sect. 6 contains comparisons and discussion of experimental data. 


\section{The Jahn-Teller Hamiltonian}

Several theoretical papers (Ceulemans and Fowler 1990, De Los Rios et al. 1996, Moateet al. 1996, Moate et al. 1997, Manini and De Los Rios 2000) formulate the $H \otimes(a+g+h)$ Jahn-Teller (JT) and dynamical JT problem - describing a hole in molecular $\mathrm{C}_{60}$ - though with different notations and conventions. For ease of comparison, it is therefore useful to set up explicitly the conventions we use in the present calculation.

The analogies of the icosahedral $H \otimes(a+g+h)$ JT coupling to a spherical $\mathcal{D}^{(2)} \otimes$ $\left(\mathcal{D}^{(0+)}+\mathcal{D}^{(2+)}+\mathcal{D}^{(4+)}\right)$ model were exploited in earlier work, where among other things it was shown that surprisingly, and depending on numbers, this dynamical JT problem may or may not possess a Berry phase (De Los Rios et al. 1996, De Los Rios and Manini 1997, Manini and De Los Rios 1998). The representations $\mathcal{D}^{(L \pm)}$ of $O(3)$ map into representations of the icosahedral group $\mathcal{I}_{h}$ as follows (Altmann and Herzig 1994): $\mathcal{D}^{(0 \pm)} \longrightarrow a_{g / u}, \mathcal{D}^{(1 \pm)} \longrightarrow t_{1 g / u}, \mathcal{D}^{(2 \pm)} \longrightarrow h_{g / u}, \mathcal{D}^{(3 \pm)} \longrightarrow t_{2 g / u} \oplus h_{g / u}$, $\mathcal{D}^{(4 \pm)} \longrightarrow g_{g / u} \oplus h_{g / u}$, and so on. This means that the decomposition of the symmetric part of the tensor product

$$
\left\{\mathcal{D}^{(2-)} \otimes \mathcal{D}^{(2-)}\right\}_{s}=\mathcal{D}^{(0+)} \oplus \mathcal{D}^{(2+)} \oplus \mathcal{D}^{(4+)}
$$

becomes in icosahedral language

$$
\left\{h_{u} \otimes h_{u}\right\}_{s}=a_{g} \oplus h_{g} \oplus\left(g_{g} \oplus h_{g}\right) \text {. }
$$

$a_{g}$ and $g_{g}$ appear only once, while the $h_{g}$ representation modes appears twice in this decomposition: one represents a genuine quadrupolar $\mathcal{D}^{(2+)}$ state, while the other one derives from a $\mathcal{D}^{(4+)}$ representation. Though this $O(3)$ picture is suggestive (Ceulemans et al. 1994), clearly a quantitative description of $\mathrm{C}_{60}^{n+}$ ions had better involve icosahedral symmetry from the beginning.

In the icosahedral group indeed in the $h_{u} \otimes h_{u}$ tensor product, the $h_{g}$ representation appears twice. This reflects the non-simple reducibility of the icosahedral symmetry group. Accordingly, Butler 1981 provides two independent sets of Clebsch-Gordan (CG) coefficients

$$
{ }^{h} C_{\mu, \nu}^{m[r]} \equiv\langle H, \mu ; H, \nu \mid h, m\rangle{ }^{[r]}
$$

which couple an $H$ electronic state (quadratically) with an $h$ vibrational mode (linearly) to give a scalar. Each set of coefficients is identified by a multiplicity index $r=1,2$. Since the two $h$ states labeled $r=1,2$ are symmetry-wise indistinguishable, the choice of these two sets of coefficients is perfectly arbitrary, as long as they are kept orthogonal to each other. This arbitrariness is the source of the different notations taken in the literature of this field. Here, we stick to Butler's choice (Butler 1981), which is basically equivalent to Ceulemans' convention (Fowler and Ceulemans 1985). Also, we label the states within a degenerate multiplet by the labels of the subgroup chain $\mathcal{I}_{h} \supset D_{5} \supset C_{5}$. For brevity, we indicate only the $C_{5}$ index $m(m=0$ 
for $a_{g}, m= \pm 1, \pm 2$ for $g_{g}$ and $m=-2, \ldots, 2$ for $h_{g}$ states) in the labeling of states since, for the representations relevant to our problem, the $D_{5}$ label is just the absolute value of $m$.

Given the tabulated CG coefficients, it is necessary for generality to consider a linear combination

$$
{ }^{h} C_{\mu, \nu}^{m}(\alpha) \equiv \cos \alpha{ }^{h} C_{\mu, \nu}^{m}{ }^{[1]}+\sin \alpha{ }^{h} C_{\mu, \nu}^{m}{ }^{[2]}
$$

of the two sets. The coefficient ${ }^{h} C_{\mu, \nu}^{m}(\alpha)$ coincides with Butler's $r=1$ and $r=2$ values for $\alpha=0$ and $\alpha=\frac{\pi}{2}$ respectively. Different values of $\alpha$ can be compared with the conventions of previous authors. For example, $\alpha=-\arctan (3 / \sqrt{5}) \approx-53.3^{\circ}$ is the case studied by De Los Rios et al. 1996 (where the $\mathcal{I}_{h}$ CG coefficient becomes equivalent to the spherical $\langle 2, \mu ; 2, \nu \mid 2, m\rangle) ; \alpha=\pi / 2$ by Moate et al. 1996; and $\alpha=0, \pi / 2$ by Ceulemans and Fowler 1990, where these cases are indicated as $h_{b}$ and $h_{a}$ respectively. The $\alpha$-dependence of these CG coefficients indicates that - unlike, for example, cubic symmetry - belonging to the $h_{g}$ group representation in icosahedral symmetry does not determine completely the form of the JT coupling. The mixing angle $\alpha$ is also needed for that. In the present case of fullerene, each $h_{g}$ distortion mode is thus characterized not only by its frequency and scalar coupling, but also by its specific mixing angle $-\pi / 2 \leq \alpha \leq \pi / 2$.

The basic linear Jahn-Teller Hamiltonian for the $H_{u} \otimes\left(a_{g}+g_{g}+h_{g}\right)$ model is conveniently divided into:

$$
H=\sum_{\tau=a_{g}, g_{g}, h_{g}} \sum_{i}^{n_{\text {modes }}(\tau)}\left[H_{\text {harm }}^{\tau}\left(\hbar \omega_{\tau i}, \vec{P}_{\tau i}, \vec{Q}_{\tau i}\right)+H_{\mathrm{e}-\mathrm{v}}^{\tau}\left(g_{\tau i} \hbar \omega_{\tau i}, \alpha_{\tau i}, \vec{Q}_{\tau i}\right)\right]
$$

The first term describes the linearly-coupled vibrations in the harmonic approximation,

$$
H_{\text {harm }}^{\tau}(\hbar \omega, \vec{P}, \vec{Q})=\frac{\hbar \omega}{2} \sum_{m}\left(P_{m}^{2}+Q_{m}^{2}\right),
$$

while the second term is the linear coupling to each mode:

$$
H_{\mathrm{e}-\mathrm{v}}^{\tau}(g \hbar \omega, \alpha, \vec{Q})=\frac{g \hbar \omega}{2} \sum_{m \mu \nu} Q_{m} c_{\mu}^{\dagger} c_{-\nu}{ }^{\tau} C_{\mu, \nu}^{m}(\alpha)
$$

Here, of course, the $\alpha$ dependence is relevant only for the $\tau=h_{g}$ case. The distortion coordinates $Q_{\tau i m}$ (with conjugate momentum $P_{\tau i m}$ ) are dimensionless, being measured in units of $x_{0}\left(\omega_{\tau i}\right)=\left(\hbar / m_{\mathrm{C}} \omega_{\tau i}\right)^{1 / 2}$. The operator $c_{\mu}^{\dagger}$ creates an electron in orbital $\mu(=-2, \ldots, 2)$ of the HOMO $H_{u}$ shell.

Naturally this form of the coupling Hamiltonian is such that each term represents pertinent irreducible representation combinations that are totally symmetrical, i.e., scalars, under the icosahedral group. For future applications, it will provide a convenient form both for perturbative calculations (small $g$ values) and as a starting point 
for numerical diagonalization methods, such as the Lanczos technique. Here, however, we restrict ourselves to a study of the classical molecular distortions. For that, it is more convenient (Manini and De Los Rios 2000) to switch to a real representation of the vibrational degrees of freedom and orbitals.

To that end, we apply two standard unitary transformations (Manini and De Los Rios 2000), one to the electronic and the other to the vibrational degrees of freedom. We define a new set of electronic operators, $d_{m}$ (and consequently their Hermitian conjugates $d_{m}^{\dagger}$ ), as

$$
\begin{aligned}
c_{0} & =d_{0} \\
\left(\begin{array}{c}
c_{m} \\
c_{-m}
\end{array}\right) & =\frac{1}{\sqrt{2}}\left(\begin{array}{cc}
1 & i \\
1 & -i
\end{array}\right)\left(\begin{array}{c}
d_{m} \\
d_{-m}
\end{array}\right), m=1,2 .
\end{aligned}
$$

This transformation leaves unchanged the (diagonal) coupling to the $a_{g}$ modes, which takes the final form:

$$
H_{\mathrm{e}-\mathrm{v}}^{a_{g}}(g \hbar \omega, q)=\frac{g \hbar \omega}{2} q \sum_{\mu \nu} d_{\mu}^{\dagger} d_{\nu} V_{\mu \nu}^{a_{g}},
$$

with $V_{\mu \nu}^{a_{g}}=\delta_{\mu \nu}$ and $q=Q_{0}$.

The second (similar) transformation is applied to the vibrational coordinates of the $g_{g}$ and $h_{g}$ modes:

$$
\begin{aligned}
Q_{0} & =q_{0} \quad\left(h_{g} \text { modes only }\right) \\
\left(\begin{array}{c}
Q_{m} \\
Q_{-m}
\end{array}\right) & =\frac{(-1)^{m}}{\sqrt{2}}\left(\begin{array}{cc}
1 & i \\
1 & -i
\end{array}\right)\left(\begin{array}{c}
q_{m} \\
q_{-m}
\end{array}\right)(m=1,2) .
\end{aligned}
$$

The harmonic part is left unchanged by this transformation, while the interaction is transformed into

$$
H_{\mathrm{e}-\mathrm{v}}^{\tau}(g \hbar \omega, \alpha, \vec{q})=\frac{g \hbar \omega}{2} \sum_{m} q_{m} \sum_{\mu \nu} d_{\mu}^{\dagger} d_{\nu} V_{\mu \nu}^{\tau(m)}(\alpha) \quad\left(\tau=g_{g}, h_{g}\right) .
$$

The $5 \times 5$ coupling matrices $\mathbf{V}^{\tau(m)}$ are combinations of the CG coefficients. Their explicit expressions [we use the shorthand $s$ for $\sqrt{3}$ and omit the explicit indication of dependence $\mathbf{V}^{h_{g}(m)}(\alpha)$ ] are the following:

$$
\begin{aligned}
\mathbf{V}^{g_{g}(-2)} & =s^{-1}\left(\begin{array}{ccccc}
0 & 0 & 0 & -\frac{1}{4} & 0 \\
0 & 0 & 0 & 1 & -\frac{1}{4} \\
0 & 0 & 0 & 0 & \frac{s}{2} \\
-\frac{1}{4} & 1 & 0 & 0 & 0 \\
0 & -\frac{1}{4} & \frac{s}{2} & 0 & 0
\end{array}\right) \\
\mathbf{V}^{g_{g}(-1)} & =s^{-1}\left(\begin{array}{ccccc}
0 & 0 & 0 & -\frac{1}{4} & -1 \\
0 & 0 & 0 & 0 & \frac{1}{4} \\
0 & 0 & 0 & -\frac{s}{2} & 0 \\
-\frac{1}{4} & 0 & -\frac{s}{2} & 0 & 0 \\
-1 & \frac{1}{4} & 0 & 0 & 0
\end{array}\right)
\end{aligned}
$$




$$
\begin{aligned}
& \mathbf{V}^{g_{g}(1)}=s^{-1}\left(\begin{array}{ccccc}
-1 & -\frac{1}{4} & 0 & 0 & 0 \\
-\frac{1}{4} & 0 & \frac{s}{2} & 0 & 0 \\
0 & \frac{s}{2} & 0 & 0 & 0 \\
0 & 0 & 0 & 0 & -\frac{1}{4} \\
0 & 0 & 0 & -\frac{1}{4} & 1
\end{array}\right) \\
& \mathbf{V}^{g_{g}(2)}=s^{-1}\left(\begin{array}{ccccc}
0 & -\frac{1}{4} & -\frac{s}{2} & 0 & 0 \\
-\frac{1}{4} & -1 & 0 & 0 & 0 \\
-\frac{s}{2} & 0 & 0 & 0 & 0 \\
0 & 0 & 0 & 1 & \frac{1}{4} \\
0 & 0 & 0 & \frac{1}{4} & 0
\end{array}\right) \\
& \mathbf{V}^{h_{g}(-2)}=\frac{\cos \alpha}{\sqrt{20}}\left(\begin{array}{ccccc}
0 & 0 & 0 & s & 0 \\
0 & 0 & 0 & s & s \\
0 & 0 & 0 & 0 & -1 \\
s & s & 0 & 0 & 0 \\
0 & s & -1 & 0 & 0
\end{array}\right)+\frac{\sin \alpha}{2 s}\left(\begin{array}{ccccc}
0 & 0 & 0 & 1 & 0 \\
0 & 0 & 0 & -1 & 1 \\
0 & 0 & 0 & 0 & s \\
1 & -1 & 0 & 0 & 0 \\
0 & 1 & s & 0 & 0
\end{array}\right) \\
& \mathbf{V}^{h_{g}(-1)}=\frac{\cos \alpha}{\sqrt{20}}\left(\begin{array}{ccccc}
0 & 0 & 0 & s & -s \\
0 & 0 & 0 & 0 & -s \\
0 & 0 & 0 & 1 & 0 \\
s & 0 & 1 & 0 & 0 \\
-s & -s & 0 & 0 & 0
\end{array}\right)+\frac{\sin \alpha}{2 s}\left(\begin{array}{ccccc}
0 & 0 & 0 & -1 & -1 \\
0 & 0 & 0 & 0 & 1 \\
0 & 0 & 0 & s & 0 \\
-1 & 0 & s & 0 & 0 \\
-1 & 1 & 0 & 0 & 0
\end{array}\right) \\
& \mathbf{V}^{h_{g}(0)}=\frac{\cos \alpha}{\sqrt{20}}\left(\begin{array}{ccccc}
1 & 0 & 0 & 0 & 0 \\
0 & 1 & 0 & 0 & 0 \\
0 & 0 & -4 & 0 & 0 \\
0 & 0 & 0 & 1 & 0 \\
0 & 0 & 0 & 0 & 1
\end{array}\right)+\frac{\sin \alpha}{2}\left(\begin{array}{ccccc}
-1 & 0 & 0 & 0 & 0 \\
0 & 1 & 0 & 0 & 0 \\
0 & 0 & 0 & 0 & 0 \\
0 & 0 & 0 & 1 & 0 \\
0 & 0 & 0 & 0 & -1
\end{array}\right) \\
& \mathbf{V}^{h_{g}(1)}=\frac{\cos \alpha}{\sqrt{20}}\left(\begin{array}{ccccc}
-s & s & 0 & 0 & 0 \\
s & 0 & -1 & 0 & 0 \\
0 & -1 & 0 & 0 & 0 \\
0 & 0 & 0 & 0 & s \\
0 & 0 & 0 & s & s
\end{array}\right)+\frac{\sin \alpha}{2 s}\left(\begin{array}{ccccc}
-1 & -1 & 0 & 0 & 0 \\
-1 & 0 & -s & 0 & 0 \\
0 & -s & 0 & 0 & 0 \\
0 & 0 & 0 & 0 & -1 \\
0 & 0 & 0 & -1 & 1
\end{array}\right) \\
& \mathbf{V}^{h_{g}(2)}=\frac{\cos \alpha}{\sqrt{20}}\left(\begin{array}{ccccc}
0 & s & 1 & 0 & 0 \\
s & -s & 0 & 0 & 0 \\
1 & 0 & 0 & 0 & 0 \\
0 & 0 & 0 & s & -s \\
0 & 0 & 0 & -s & 0
\end{array}\right)+\frac{\sin \alpha}{2 s}\left(\begin{array}{ccccc}
0 & 1 & -s & 0 & 0 \\
1 & 1 & 0 & 0 & 0 \\
-s & 0 & 0 & 0 & 0 \\
0 & 0 & 0 & -1 & -1 \\
0 & 0 & 0 & -1 & 0
\end{array}\right) \text {. }
\end{aligned}
$$

In the static JT effect, the kinetic term in $P_{\text {rim }}^{2}$ is ignored, and the problem is to study the five Born-Oppenheimer (BO) potential sheets given by the sum of each eigenvalue of the electronic problem plus the harmonic restoring forces. The $H_{u} \otimes$ $a_{g}$ part has a purely diagonal coupling matrix. As it does not split the electronic degeneracy, it is trivially separated from the coupling to the other modes and can be treated as a displaced oscillator. 
When a single electron (hole) is placed in the $H_{u}$ orbital, the molecule distorts in such a way that the lowest (highest) BO sheet is lowered (raised) in energy as much as possible. The coupling to a $g_{g}$ mode leads to 10 equivalent absolute minima of $D_{3}$ local symmetry (Ceulemans and Fowler 1990, Manini and De Los Rios 2000) of the BO potential. The optimal distortion is realized for $\left|q_{\mathrm{s}}^{g_{g}}\right|=g_{g_{g}} / 3$, with a corresponding potential energy lowering of $g_{g_{g}}^{2} \hbar \omega_{g_{g}} / 18$. The $r=2$ part of the coupling to the $h_{g}$ modes (corresponding to the $\sin \alpha$ terms in $\mathbf{V}^{h_{g}(m)}$ above) contributes to these same minima in an equivalent way, with $g_{g_{g}}$ replaced by $g_{h_{g}} \sin \alpha$. However, the $r=1$ component ( $\cos \alpha$ terms in $\mathbf{V}^{h_{g}(m)}$ ) of the coupling favours the six classical stable minima of local $D_{5}$ symmetry (Ceulemans and Fowler 1990, Manini and De Los Rios 2000). The optimal distortion at these minima is $\left|q_{\mathrm{s}}^{h_{g}}\right|=g_{h_{g}} \cos \alpha / \sqrt{5}$, for an energy lowering of $g_{h_{g}}^{2} \cos ^{2} \alpha \hbar \omega / 10$.

The simultaneous linear coupling to several modes will generally lead to a cumulative distortion and to an energy gain which is the sum of the individual energy gains. However, the form of the coupling (11) prevents the molecule to gain energy through both kinds of couplings. The system shall choose between a $D_{3}$ and a $D_{5}$ distortion, depending which one is energetically more convenient for given specific values of the couplings, vibration frequencies, and $H_{u}$ orbital electronic filling. The calculation of the following section determines in particular which one of the two types of distortions prevails in $\mathrm{C}_{60}^{+}$.

\section{Calculation of the couplings and results}

We compute the molecular electronic structure within the density functional theory (DFT) in the local density approximation. The $\mathrm{C}_{60}$ molecule is repeated periodically in a large fcc supercell lattice. The conventional supercell side is $a=18.5 \AA$, so that the distance between the centers of two neighboring copies of the molecule is $13.1 \AA$, suitably much larger than the fully relaxed equilibrium (opposite C-C) ball diameter, about $7.053 \AA$. Since we aim at describing the single molecule - and indeed our molecules are well isolated - no sampling of the Brillouin zone is called for, and calculations of the charge density are done using the $k=0$ wavefunctions. We use ultrasoft pseudopotentials (Vanderbilt 1990) for C (Favot and Dal Corso 1999). The plane-waves basis set is cut off at $E_{\text {cut }}=27 \mathrm{Ry}$ (charge density cutoff $=162 \mathrm{Ry}$ ). Test calculations with higher cutoff or larger cell size $a$ gave equivalent results.

Based on this electronic structure calculation, we used next density functional perturbation theory (Baroni et al. 1987) to compute three independent rows of the dynamical matrix. Icosahedral symmetry is then used to recover the full matrix, which determines the normal modes $\vec{\xi}_{i, s}$ and frequencies $\omega_{i}$ (Giannozzi and Baroni 1994) of the molecule. We obtained frequencies (see Table 1) in good agreement with experiment (Prassides et al. 1991, Zhou et al. 1992), as well as with previous calculations (Giannozzi and Baroni 1994, Negri et al. 1988, Kohanoff et al. 1992). 


\begin{tabular}{lrrr}
\hline \hline mode & Experim. & Giannozzi \& Baroni & this work \\
\hline$a_{g}(1)$ & 496 & 495 & 500 \\
$a_{g}(2)$ & 1470 & 1504 & 1511 \\
\hline$h_{g}(1)$ & 271 & 259 & 261 \\
$h_{g}(2)$ & 437 & 425 & 429 \\
$h_{g}(3)$ & 710 & 711 & 718 \\
$h_{g}(4)$ & 774 & 783 & 784 \\
$h_{g}(5)$ & 1099 & 1120 & 1119 \\
$h_{g}(6)$ & 1250 & 1281 & 1275 \\
$h_{g}(7)$ & 1428 & 1450 & 1456 \\
$h_{g}(8)$ & 1575 & 1578 & 1588 \\
\hline$t_{1 u}(1)$ & 527 & 527 & 533 \\
$t_{1 u}(2)$ & 576 & 586 & 588 \\
$t_{1 u}(3)$ & 1183 & 1218 & 1212 \\
$t_{1 u}(4)$ & 1428 & 1462 & 1469 \\
\hline \hline
\end{tabular}

Table 1: Eigenfrequencies (in $\mathrm{cm}^{-1}$ ) for the Raman- and IR-active vibrational modes of $\mathrm{C}_{60}$ molecule: comparisons with experimental values (Prassides et al. 1991, Zhou et al. 1992, Gunnarsson et al. 1995) and previous calculation (Giannozzi and Baroni 1994).

To determine the e-v couplings for the linearly coupled modes, we proceed subsequently to displace the atomic positions from the equilibrium position along each of the normal modes, choosing a suitable eigenvector combination in the linear space of each degenerate vibration. In particular for each $h_{g}$ mode we selected the $q_{0}$ displacement, corresponding to the totally symmetric combination $\vec{\xi}_{i, 0}$ of the distortions $\vec{\xi}_{i, s}$ with respect to an (arbitrarily chosen) $D_{5}$ subgroup of the molecular symmetry group. The five initially degenerate (really, only nearly degenerate, owing to a weak cubic splitting due to the artificial supercell lattice) $H_{u}$ Kohn-Sham eigenvalues split under this distortion with a pattern given by the eigenvalues of $\mathbf{V}^{h_{g}(0)}$. We applied a displacement of the atomic positions along each of the eight normal-mode unit vectors, $\vec{\xi}_{i, 0}$ with a prefactor ranging from -0.1 to $0.1 \AA$. In Fig. 1 we plot as an example the resulting energies for the sixth $h_{g}$ mode. The pattern generated by $\mathbf{V}^{h_{g}(0)}$ should be $1+2+2$ (a state separated by two pairs of twofold-degenerate states). The small residual splittings of these twofold degeneracies, due to the cubic crystal field and higher-than-linear couplings, give an estimate of the accuracy of the method. By standard linear fitting and comparison with Eq. (11), we obtained directly the linear dimensionless coupling coefficients $g_{h_{g} i} \alpha_{h_{g} i}$ collected in Table 2. We determined the sign of $\alpha_{h_{g} i}$ by applying a distortion along $\vec{\xi}_{i, 1}$, and comparing the splitting of the HOMO with the eigenvalues of $\mathbf{V}^{h_{g}(1)}\left( \pm \alpha_{h_{g} i}\right)$. Following the same procedure we derived the couplings for the $g_{g}$ modes, by applying here $q_{-1}$ distortions. The resulting 


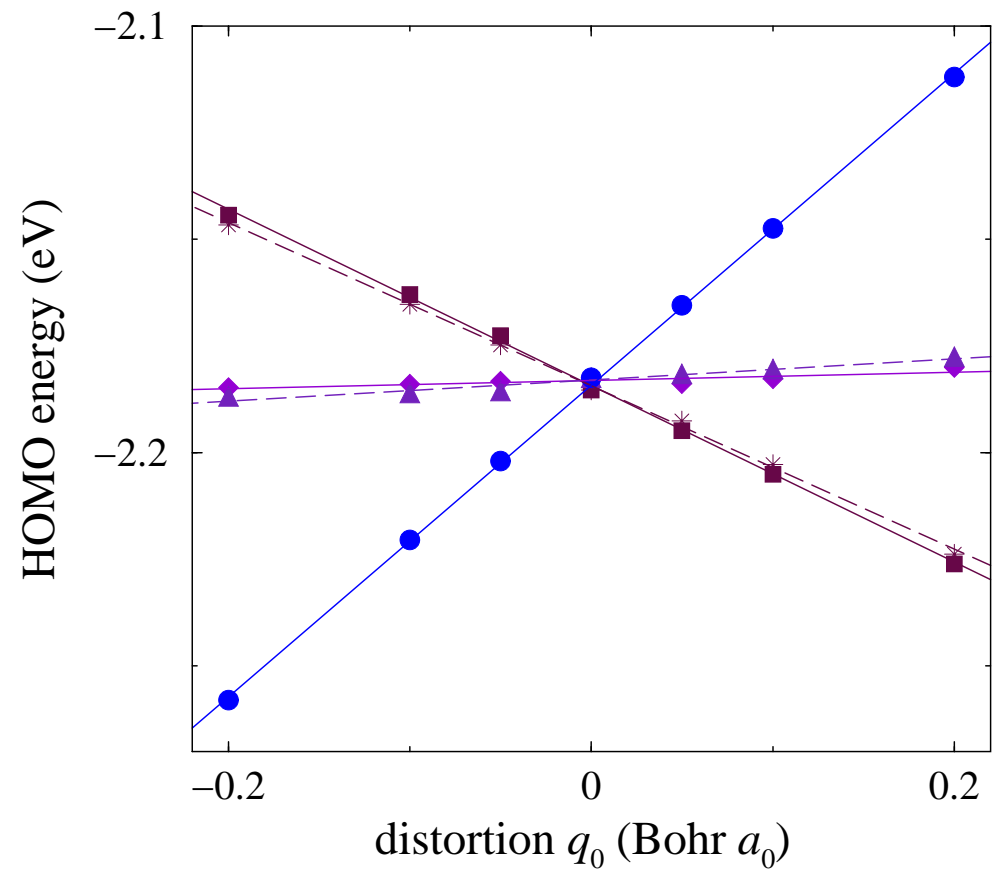

Figure 1: The splitting of the HOMO degenerate level when the molecule is distorted according to the 6th mode $h_{g}\left(1250 \mathrm{~cm}^{-1}\right)$. Points: DFT data. Lines: linear fits, giving the coupling parameters $g_{h_{g} 6}$ and $\alpha_{h_{g} 6}$ of Table 2 .

linear coupling coefficients $g_{g_{g} i}$ are also collected in Table 2 .

For convenience, we also report in Table 2 the amount of optimal JT distortion pertinent to each mode when the HOMO level is occupied by one electron/hole, and the corresponding energy lowering $E_{\mathrm{s}}$ for both $D_{5}$ and $D_{3}$ minima. Note in particular the large coupling associated to the lowest $h_{g}$ mode, the corresponding distortion leading to an energy lowering practically equal to its quantum $\hbar \omega$.

Adding up the JT energy gain of the individual modes, we estimate the total classical potential energy lowering in $\mathrm{C}_{60}$. The $D_{5}$ minima gain $E_{\mathrm{s}}=71 \mathrm{meV}$, while the $D_{3}$ minima gain only $E_{\mathrm{s}}=22 \mathrm{meV}$ (the contribution of the $a_{g}$ modes $-2 \mathrm{meV}-$ being included in both cases). It is therefore apparent that the $\mathrm{C}_{60}^{+}$ion will choose, at least within linear coupling, the $D_{5}$ distortion. As was shown in (Manini and De Los Rios 2000), the possibility of a switch to a nondegenerate $A_{u}$ dynamical JT GS occurs, for large coupling strength $g \geq 6$, only under the condition that the $D_{3}$ minima are energetically lower or equal to the $D_{5}$ minima. This settles finally the issue of the dynamical JT GS symmetry of this molecular ion: it is a regular Berryphase vibronic state of symmetry $H_{u}$, like the parent electronic state (Manini and De Los Rios 2000). No level crossing to a nondegenerate $A_{u}$ state is predicted to occur for $\mathrm{C}_{60}^{+}$.

A static JT coupling resolves the degeneracy of the $H_{u}$ level: in a distorted config- 


\begin{tabular}{rcccccccr}
\hline \hline $\begin{array}{r}\hbar \omega_{\tau i} \\
\mathrm{~cm}^{-1}\end{array}$ & $\begin{array}{c}\text { coupl. } \\
\mathrm{eV} / a_{0}\end{array}$ & $g_{\tau i}$ & $\begin{array}{c}\alpha_{\tau i} \\
\mathrm{deg}\end{array}$ & $\begin{array}{c}q_{\mathrm{s}}\left(D_{5}\right) \\
a_{0}\end{array}$ & $\begin{array}{c}q_{\mathrm{s}}\left(D_{3}\right) \\
a_{0}\end{array}$ & $\begin{array}{c}E_{\mathrm{s}}\left(D_{5}\right) \\
\mathrm{meV}\end{array}$ & $\begin{array}{c}E_{\mathrm{s}}\left(D_{3}\right) \\
\mathrm{meV}\end{array}$ & $\begin{array}{c}\tilde{\lambda} \\
\mathrm{meV}\end{array}$ \\
\hline$a_{g}$ & & & & & & & & \\
500 & 0.03 & 0.059 & - & 0.00 & 0.00 & 0.0 & 0.0 & 0.1 \\
1511 & 0.63 & 0.274 & - & 0.01 & 0.01 & 1.8 & 1.8 & 3.5 \\
\hline$g_{g}$ & & & & & & & & \\
483 & 0.31 & 0.757 & - & - & 0.04 & 0.0 & 1.9 & 8.6 \\
567 & 0.05 & 0.102 & - & - & 0.00 & 0.0 & 0.0 & 0.2 \\
772 & 0.67 & 0.800 & - & - & 0.03 & 0.0 & 3.4 & 15.3 \\
1111 & 0.90 & 0.624 & - & - & 0.02 & 0.0 & 3.0 & 13.4 \\
1322 & 0.43 & 0.228 & - & - & 0.01 & 0.0 & 0.5 & 2.1 \\
1519 & 1.08 & 0.467 & - & - & 0.01 & 0.0 & 2.3 & 10.3 \\
\hline$h_{g}$ & & & & & & & & \\
261 & 0.50 & 3.042 & -0.1 & 0.27 & 0.00 & 30.0 & 0.0 & 75.0 \\
429 & 0.43 & 1.223 & 30.1 & 0.07 & 0.03 & 6.0 & 1.1 & 19.9 \\
718 & 0.75 & 0.995 & 89.4 & 0.00 & 0.04 & 0.0 & 4.9 & 22.0 \\
785 & 0.67 & 0.784 & -2.3 & 0.04 & 0.00 & 6.0 & 0.0 & 15.0 \\
1119 & 0.32 & 0.221 & 76.6 & 0.00 & 0.01 & 0.0 & 0.4 & 1.7 \\
1275 & 0.93 & 0.519 & 28.0 & 0.02 & 0.01 & 3.3 & 0.5 & 10.7 \\
1456 & 2.09 & 0.962 & 28.1 & 0.03 & 0.01 & 13.0 & 2.1 & 41.7 \\
1588 & 2.15 & 0.869 & -31.1 & 0.03 & 0.01 & 10.9 & 2.2 & 37.1 \\
\hline \hline
\end{tabular}

Table 2: Computed mode eigenfrequencies and e-v linear coupling parameters of the $H_{u} \mathrm{HOMO}$ in $\mathrm{C}_{60}$. The $\mathrm{JT}$ distortion magnitudes $q_{\mathrm{s}}$ and the classical stabilization energies $E_{\mathrm{s}}$ are tabulated for both $D_{5}$ and $D_{3}$ distortions and for one hole in the HOMO. The largest total JT energy gain is realized by the $D_{5}$ distortions. We also show the contribution of each mode to the resistivity and superconductivity total coupling $\tilde{\lambda}=\lambda / N_{1}(0)$ defined in Eq. (20) $\left[N_{1}(0)=\right.$ density of states per spin per band at the Fermi level].

uration it is possible to distinguish individual levels within the HOMO, energy-wise. Figure 2 depicts the square modulus of the hole wavefunction for a $D_{5}$ minimum. The probability density appears to be localized on an equatorial conjugated band, where the poles are the opposite pentagons centered around the $D_{5}$ axis we chose among the six possible ones.

As a check, with the same method used above to calculate the hole-vibration couplings of the HOMO (Fig. 1), we also computed the electron-vibration couplings of the LUMO and obtained the values in Table 3. The total static JT potential energy lowering is $41 \mathrm{meV}$, of which $3 \mathrm{meV}$ due to the $a_{g}$ modes, and $38 \mathrm{meV}$ due to the $h_{g}$ modes. These values are generally in line with those calculated by previous authors (Antropov et al. 1993, Varma et al. 1991, Lannoo et al. 1991), although there 


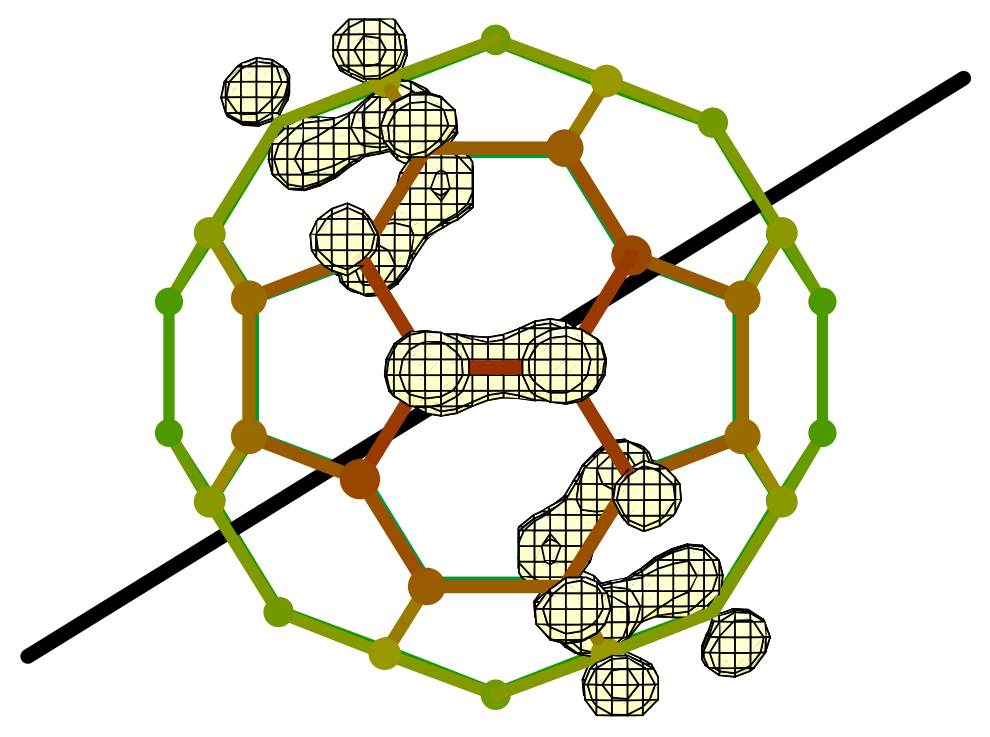

Figure 2: Electronic charge density distribution of the hole in $\mathrm{C}_{60}^{+}$at the $D_{5} \mathrm{JT}$ minimum along a $q_{0}$ distortion of any $h_{g}$ mode. Out of the six equivalent ones, this particular minimum is defined by the $D_{5}$ axis drawn as a bold "spit" piercing the ball through two pentagons. The contours are drawn at $30 \%$ of the maximum density.

are some differences in the details. Error bars in theoretical determinations of JT couplings of fullerene have proven surprisingly large, possibly reflecting and amplifying errors in the vibrational eigenvectors. Of course as is well known, somewhat larger energy gains are obtained when the true dynamical JT problem, including a full quantum treatment of the vibrons is considered (Auerbach et al. 1994, Manini et al. 1994). We shall leave this calculation in $\mathrm{C}_{60}^{n+}$ for future work.

When more than one electron/hole occupies the HOMO of $\mathrm{C}_{60}$, larger distortions and more important energy gains are expected. In particular, the $a_{g}$ contribution for $\mathrm{C}_{60}^{n+}$ is simply:

$$
E_{\mathrm{s}}^{a_{g}}(n)=n^{2} E_{\mathrm{s}}^{a_{g}}(1),
$$

which can become as large as $179 \mathrm{meV}$ for $n=10$ holes. Consider now the nontrivial JT part of the coupling, that to $g_{g}$ and $h_{g}$ modes. For $n=2$ electrons/holes (spin singlet configuration) in the HOMO orbital, the distortions simply become twice as large as in the $n=1$ case, with JT energy gains which are four times larger. Even though $n=1,2$ electrons/holes take advantage only of the $D_{5}$ stabilization energy, additional electrons/holes can benefit from the extra HOMO splitting induced by the $h_{g}(r=2)$ plus $g_{g}$ coupling. To test this, we relaxed completely the molecular structure in the 64-dimensional space of the $8 h_{g}$ and $g_{g}$ modes, and determined the minima of the total potential energy, filling the five $\mathrm{BO}$ sheets as drawn in the insets of Fig. 3 . For simplicity we only considered at this stage low-spin configurations, generally the most favored by JT. The resulting energy gains $E_{\mathrm{s}}^{g_{g}+h_{g}}(n)$ are reported in Fig. 3. 


\begin{tabular}{rccccc}
\hline \hline $\begin{array}{r}\hbar \omega_{\tau i} \\
\mathrm{~cm}^{-1}\end{array}$ & $\begin{array}{c}\text { coupl. } \\
\mathrm{eV} / a_{0}\end{array}$ & $g_{\tau i}$ & $\begin{array}{c}q_{\mathrm{s}} \\
a_{0}\end{array}$ & $\begin{array}{c}E_{\mathrm{s}} \\
\mathrm{meV}\end{array}$ & $\begin{array}{c}\tilde{\lambda} \\
\mathrm{meV}\end{array}$ \\
\hline$a_{g}$ & & & & & \\
500 & 0.07 & 0.157 & 0.01 & 0.2 & 0.4 \\
1511 & 0.78 & 0.340 & 0.01 & 2.7 & 5.4 \\
\hline$h_{g}$ & & & & & \\
261 & 0.07 & 0.412 & 0.08 & 2.7 & 13.7 \\
429 & 0.17 & 0.489 & 0.07 & 6.3 & 31.7 \\
718 & 0.26 & 0.350 & 0.04 & 5.5 & 27.3 \\
785 & 0.19 & 0.224 & 0.03 & 2.4 & 12.2 \\
1119 & 0.28 & 0.193 & 0.02 & 2.6 & 12.9 \\
1275 & 0.25 & 0.138 & 0.01 & 1.5 & 7.6 \\
1456 & 0.69 & 0.315 & 0.03 & 9.0 & 44.8 \\
1588 & 0.72 & 0.289 & 0.02 & 8.2 & 41.2 \\
\hline \hline
\end{tabular}

Table 3: Computed mode eigenfrequencies and e-v linear coupling parameters for the $T_{1 u}$ LUMO of $\mathrm{C}_{60}$. For each mode, we report The JT distortion magnitudes $q_{\mathrm{s}}$, the classical stabilization energies $E_{\mathrm{s}}$, and the contribution to $\tilde{\lambda}=\lambda / N_{1}[0]$.

The energy lowering is maximum for $n=4$ (and $n=6$ ): it is as large as $401 \mathrm{meV}$, compared to a modest $E_{\mathrm{s}}^{h_{g}}(n=2,4)=153 \mathrm{meV}$ in $\mathrm{C}_{60}^{-}$. Similarly to what happens in the $T_{1 u}$ LUMO case (see Fig. 3 and Manini et al. 1994), the half-filled configuration $n=5$ is slightly unfavorable (by JT energetics) with respect to the neighboring $n=4$ and $n=6$ states. We find that the contributions of the $g_{g}$ modes, strictly zero for $n=1,2$, are small $(\sim 1 \mathrm{meV})$ but nonzero in the $3 \leq n \leq 7$ configurations. This indicates that for such large fillings the many-modes JT system (Manini and Tosatti 1998) can take some advantage also of the "losing" $g_{g}+h_{g}(r=2)$ part of the coupling (favoring $D_{3}$ minima for $n=1,2$ ). The insets of Fig. 3, indicate that the largest displacement of a single level in the $H_{u} \mathrm{HOMO}$, about $0.28 \mathrm{eV}$, is realized for $n=2$. Finally, we have particle-hole symmetry $E_{\mathrm{s}}^{g_{g}+h_{g}}(n)=E_{\mathrm{s}}^{g_{g}+h_{g}}(10-n)$, at an opposite minimum distortion $\vec{q}_{\mathrm{s}}(n)=-\vec{q}_{\mathrm{s}}(10-n)$. For the special case $n=5$ this means that the configuration drawn in the inset and the one obtained reflecting the 5 levels through zero give both the same optimal energy (at opposite distortions).

Application of the above results to real $\mathrm{C}_{60}^{n+}$ must await the inclusion of electronelectron Coulomb repulsion. Coulomb interactions will generally compete with JT coupling and favor high-spin configurations, which, in turn, are generally less favorable for JT. (For example for $n=2$ the triplet configurations has a JT gain of $100 \mathrm{meV}$ instead of $277 \mathrm{meV}$ for the singlet.) We shall return to a more detailed description of $\mathrm{C}_{60}^{n+}$ ions in later work. 


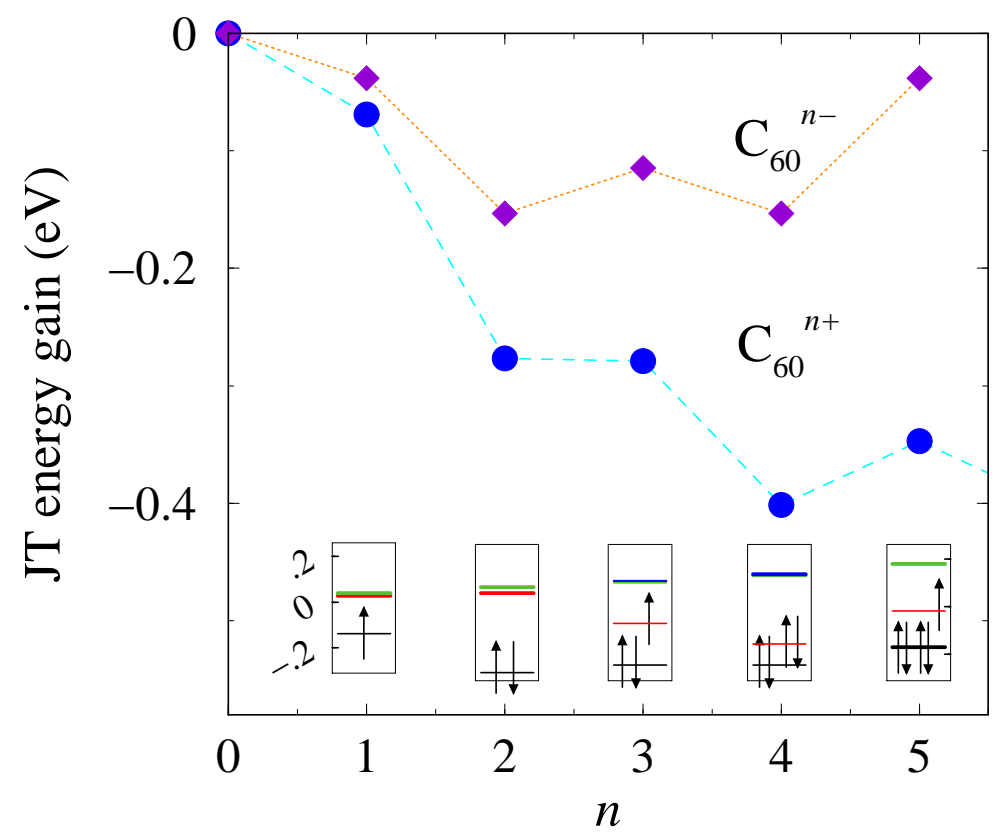

Figure 3: The static JT energy gain (excluding the $a_{g}$ modes contribution) of $\mathrm{C}_{60}^{n+}$, as a function of the filling $n$ of the $H_{u}$ molecular level. Electron-hole symmetrical values are obtained beyond half-filling $n=5$. The corresponding energies for the $T_{1 u}$ LUMO $\left(\mathrm{C}_{60}^{n-}\right)$ are also plotted for comparison. Note the much larger gains (up to a factor 3) in the $H_{u}$ case. For each $n$, the insets show the corresponding split HOMO electronic configuration (all in the same scale of eV).

\section{Solid-State Transport}

In order to relate the previously calculated electron-vibron coupling constants to relevant solid state physical quantities, we start by re-deriving its contribution to the transport relaxation time. We are interested in particular to the features of an orbitally degenerate band as in the charged $\mathrm{C}_{60}$ case. The Boltzmann equation in the presence of a uniform and static electric field $\vec{E}$ reads

$$
e \vec{v}_{k \mu} \cdot \vec{E} \frac{\partial n^{0}\left(\epsilon_{k \mu}\right)}{\partial \epsilon_{k \mu}}=\left(\frac{\partial n_{k, \mu}}{\partial t}\right)_{\text {coll }}
$$

being $n_{k, \mu}$ and $\epsilon_{k \mu}$ the occupation number and energy at momentum $k$ for orbital $\mu$, and $\vec{v}_{k \mu}=\partial \epsilon_{k \mu} / \partial \vec{k}$.

Within the relaxation time approximation, $\partial n_{k, \mu} / \partial t=\delta n_{k, \mu} / \tau_{k \mu}$ so that the variation with respect to equilibrium becomes

$$
\delta n_{k, \mu}=-\frac{\partial n^{0}\left(\epsilon_{k \mu}\right)}{\partial \epsilon_{k \mu}} e \vec{v}_{k \mu} \cdot \vec{E} \tau_{k \mu}
$$


and the conductivity is obtained in the form

$$
\sigma=-e^{2} \frac{1}{3 V} \sum_{k \mu} \tau_{k \mu} \vec{v}_{k \mu} \cdot \vec{v}_{k \mu} \frac{\partial n^{0}\left(\epsilon_{k \mu}\right)}{\partial \epsilon_{k \mu}},
$$

$V$ being the volume, measured in cell units.

As is usual in the treatment of different scattering mechanisms within Fermi's golden rule approximation, one has to sum the inverse of the corresponding relaxation times. The collision term due to the electron-vibron coupling is given, within Fermi's golden rule, by

$$
\begin{aligned}
& \left(\frac{\partial n_{k, \mu}}{\partial t}\right)_{c o l l}=\frac{2 \pi}{\hbar} \frac{1}{2 V} \sum_{q} \sum_{i, \tau, m, \nu}\left(\frac{g_{\tau i} \hbar \omega_{\tau i}}{2}\right)^{2}\left|V_{\nu \mu}^{\tau(m)}\left(\alpha_{\tau i}\right)\right|^{2}\{ \\
& n_{k-q, \nu}\left(1-n_{k, \mu}\right)\left[N_{q, m}^{\tau i} \delta\left(\epsilon_{k \mu}-\epsilon_{k-q \nu}-\hbar \omega_{\tau i}\right)+\left(N_{-q, m}^{\tau i}+1\right) \delta\left(\epsilon_{k \mu}-\epsilon_{k-q \nu}+\hbar \omega_{\tau i}\right)\right] \\
& \left.-n_{k, \mu}\left(1-n_{k-q, \nu}\right)\left[N_{-q, m}^{\tau i} \delta\left(\epsilon_{k-q \nu}-\epsilon_{k \mu}-\hbar \omega_{\tau i}\right)+\left(N_{q, m}^{\tau i}+1\right) \delta\left(\epsilon_{k-q \nu}-\epsilon_{k \mu}+\hbar \omega_{\tau i}\right)\right]\right\} .
\end{aligned}
$$

Here $N_{q, m}^{\tau i}$ is the Bose-Einstein occupation number of phonons of crystal momentum $q$, symmetry $\tau=a_{g}, g_{g}, h_{g}$, mode $i$, component $m$. $n_{k, \mu}$ is the Fermi occupation of HOMO band component $\mu=-2, \ldots, 2$ at the given temperature and chemical potential. At equilibrium, the collision term is zero. We expand to first order in the deviation from equilibrium.

First, let us consider the case $T \gg \operatorname{Max}_{i}\left(\hbar \omega_{i}\right)$ but still $T \ll T_{F}$ (the Fermi temperature). In this case

$$
N_{q, m}^{\tau i} \simeq \frac{k_{\mathrm{B}} T}{\hbar \omega_{\tau i}} \gg 1
$$

and

$$
\begin{aligned}
& \left(\frac{\partial n_{k, \mu}}{\partial t}\right)_{\text {coll }}=-\frac{2 \pi}{\hbar} \frac{1}{2 V} \sum_{p} \sum_{i, \tau, m, \nu}\left(\frac{g_{\tau i} \hbar \omega_{\tau i}}{2}\right)^{2}\left|V_{\nu \mu}^{\tau(m)}\left(\alpha_{\tau i}\right)\right|^{2} \frac{k_{\mathrm{B}} T}{\hbar \omega_{\tau i}}\{ \\
& \left.\left(\delta n_{k, \mu}-\delta n_{p, \nu}\right)\left[\delta\left(\epsilon_{k \mu}-\epsilon_{p \nu}-\hbar \omega_{\tau i}\right)+\delta\left(\epsilon_{k \mu}-\epsilon_{p \nu}+\hbar \omega_{\tau i}\right)\right]\right\} .
\end{aligned}
$$

Since $T \gg \operatorname{Max}_{i}\left(\hbar \omega_{i}\right)$, after inserting (14) into (17) and using the Boltzmann equation, we find that

$$
\begin{aligned}
\frac{1}{\tau_{k \mu}}= & \frac{2 \pi}{\hbar} \frac{1}{2 V} \sum_{p} \sum_{i, \tau, m, \nu}\left(\frac{g_{\tau i} \hbar \omega_{\tau i}}{2}\right)^{2}\left|V_{\nu \mu}^{\tau(m)}\left(\alpha_{\tau i}\right)\right|^{2} \frac{k_{\mathrm{B}} T}{\hbar \omega_{\tau i}}\{ \\
& \left.\left(1-\frac{\vec{v}_{p \nu} \cdot \vec{n}}{\vec{v}_{k \mu} \cdot \vec{n}}\right)\left[\delta\left(\epsilon_{k \mu}-\epsilon_{p \nu}-\hbar \omega_{\tau i}\right)+\delta\left(\epsilon_{k \mu}-\epsilon_{p \nu}+\hbar \omega_{\tau i}\right)\right]\right\},
\end{aligned}
$$

where $\vec{n}$ is a unit vector parallel to the electric field. 
If we approximately take $\epsilon_{k \mu}=\epsilon_{k}$ we find, since the electron-vibron matrices are symmetrical,

$$
\begin{aligned}
\frac{1}{\tau_{k}}= & \frac{\pi}{\hbar} \sum_{i, \tau, m}\left(\frac{g_{\tau i} \hbar \omega_{\tau i}}{2}\right)^{2} \frac{\operatorname{Tr}\left[\left(\mathbf{V}^{\tau}\right)^{2}\right]}{d} \frac{k_{\mathrm{B}} T}{\hbar \omega_{\tau i}}\{ \\
& \left.\frac{1}{V} \sum_{p}\left(1-\frac{\vec{v}_{p} \cdot \vec{n}}{\vec{v}_{k} \cdot \vec{n}}\right)\left[\delta\left(\epsilon_{k}-\epsilon_{p}-\hbar \omega_{\tau i}\right)+\delta\left(\epsilon_{k}-\epsilon_{p}+\hbar \omega_{\tau i}\right)\right]\right\} .
\end{aligned}
$$

where $d$ is the orbital multiplicity, 5 for the HOMO-derived band of $\mathrm{C}_{60}$. Therefore, the relaxation rate in the linear $T$ regime turns out to be roughly proportional to

$$
\tilde{\lambda}=\sum_{i, \tau, m} \frac{g_{\tau i}^{2} \hbar \omega_{\tau i}}{4} \frac{\operatorname{Tr}\left[\left(\mathbf{V}^{\tau}\right)^{2}\right]}{d}
$$

related to the conventional dimensionless ep-phonon coupling $\lambda$ by

$$
\lambda=N_{1}\left(\epsilon_{F}\right) \tilde{\lambda},
$$

where $N_{1}\left(\epsilon_{F}\right)$ is the density of states per spin per band at the Fermi energy.

By using Eq. (20) and the calculated e-v coupling parameters of Table 2, we find for holes in the $H_{u} \mathrm{HOMO}$-derived band

$$
\tilde{\lambda}_{+} \simeq 0.277 \mathrm{eV}
$$

This value can be compared with that calculated similarly, using our couplings of Table 3 for electrons in the $T_{1 u}$ band

$$
\tilde{\lambda}_{-} \simeq 0.197 \mathrm{eV}
$$

We conclude therefore that holes have a stronger scattering with intra-molecular optical phonons than electrons, by about a factor 1.4 .

To confirm the accuracy of the values computed for the HOMO case, it is useful to compare those for the LUMO with previous similar calculations. It is then necessary to take into account the factor $d=3$ which is usually incorporated in the total density of states (Lannoo et al. 1991). Our $\tilde{\lambda} / d=66 \mathrm{meV}$ compares well with previous similar calculations for the $\mathrm{C}_{60}$ LUMO (Antropov et al. 1993, Varma et al. 1991, Lannoo et al. 1991), ranging from 49 to $68 \mathrm{meV}$. On the other hand, empirical estimates based on a fit to photoemission data tend to give a value as large as $147 \mathrm{meV}$ (Gunnarsson et al. 1995). The origin of this discrepancy is presently unclear.

\section{Superconductivity}

The dimensionless electron-vibron coupling which governs the transport properties, $\lambda$ of Eqs. (20,21), is not in point of principle coincident with the parameter $\lambda$ determining the superconducting properties. The latter must be determined by solving the 
Migdal-Eliashberg equation with the retarded interaction mediated by the vibrons plus the electron-electron Coulomb repulsion. However, a simple estimate of the order of magnitude of $\lambda$ can be obtained by taking the unretarded limit, and imposing a Debye cutoff to the electron energies. We assume the electronic band operators to be related to the molecular creation and annihilation operators through the unitary (orthogonal) transformation

$$
c_{n k \sigma}=U_{n \mu}^{-1}(k) d_{\mu k \sigma}
$$

$n$ being the band index, $\sigma$ the spin. Let us define a matrix $\mathbf{W}$ by

$$
W_{\mu \nu} \equiv \sum_{\tau, i, \widehat{m}} \frac{g_{\tau i}^{2} \hbar \omega_{\tau i}}{4}\left(V_{\mu \nu}^{\tau(\widehat{m})}\right)^{2}=\sum_{\tau} W_{\mu \nu}^{\tau} \sum_{i=1}^{n_{m}(\tau)} \frac{g_{\tau i}^{2} \hbar \omega_{\tau i}}{4}
$$

as well as

$$
\mathcal{W}_{n k, m p} \equiv \sum_{\tau, i, \widehat{m}} \sum_{\mu_{1}, \mu_{2}, \mu_{3}, \mu_{4}} \frac{g_{\tau i}^{2} \hbar \omega_{\tau i}}{4} U_{n \mu_{1}}^{-1}(k) V_{\mu_{1} \mu_{2}}^{\tau(\widehat{m})} U_{\mu_{2} m}(-p) U_{\mu_{3} m}(p) V_{\mu_{4} \mu_{3}}^{\tau(\widehat{m})} U_{n \mu_{4}}^{-1}(-k),
$$

The Bardeen-Cooper-Schrieffer (BCS) gap equation for

$$
\Delta_{k, n}=\left\langle c_{k, \uparrow, n}^{\dagger} c_{-k, \downarrow, n}^{\dagger}\right\rangle,
$$

reads

$$
\Delta_{k, n}=\frac{1}{2 V} \sum_{p, m} \mathcal{W}_{n k, m p} \frac{\Delta_{p, m}}{E_{p, m}} \tanh \left(\beta \frac{E_{p, m}}{2}\right)
$$

where

$$
E_{p, m}=\sqrt{\left(\epsilon_{p m}-\mu_{0}\right)^{2}+\Delta_{p, m}^{2}}
$$

$\mu_{0}$ being the chemical potential. The critical temperature is obtained by solving the eigenvalue equation

$$
\delta_{n m} \delta_{k p}-\frac{1}{2 V} \sum_{p, m} \mathcal{W}_{n k, m p} \frac{1}{\left|\epsilon_{p m}-\mu_{0}\right|} \tanh \left(\beta_{c} \frac{\left|\epsilon_{p m}-\mu_{0}\right|}{2}\right)=0 .
$$

In general, the BCS gap equation (26) leads to interference between the Cooper pairs belonging to different bands. That, in turn, increases the critical temperature relative to a situation in which the pairs do not interfere. We may therefore foresee two opposite limits of strongly interfering and of non interfering pairs which, respectively, over and underestimate the effective coupling strength $\lambda$.

If we assume that averages over the Fermi surface do not depend on the band indices (interfering pairs, corresponding, for example, to the choice of Lannoo et al. 1991), then we can replace $\mathcal{W}$ with $\mathbf{W}$, and we find that the critical temperature is 
determined by the maximum eigenvalue of the matrix $\mathbf{W}$ in Eq. (24). Under this assumption, the superconducting $\lambda$ is determined through

$$
\lambda=N_{1}\left(\epsilon_{F}\right) \operatorname{Max}_{\text {eigenvalue }}(\mathbf{W}),
$$

For our $H \otimes(a+g+h)$ e-ph coupling the matrices $\mathbf{W}^{\tau}$ are:

$$
\begin{aligned}
& \mathbf{W}^{g_{g}}=\frac{1}{24}\left(\begin{array}{ccccc}
8 & 1 & 6 & 1 & 8 \\
1 & 8 & 6 & 8 & 1 \\
6 & 6 & 0 & 6 & 6 \\
1 & 8 & 6 & 8 & 1 \\
8 & 1 & 6 & 1 & 8
\end{array}\right) \\
& \mathbf{W}^{h_{g}}= \frac{1}{60}\left(\begin{array}{ccccc}
16 & 14 & 9 & 14 & 7 \\
14 & 16 & 9 & 7 & 14 \\
9 & 9 & 24 & 9 & 9 \\
14 & 7 & 9 & 16 & 14 \\
7 & 14 & 9 & 14 & 16
\end{array}\right) \\
&+\frac{\cos (2 \alpha)}{30}\left(\begin{array}{ccccc}
-2 & 2 & -3 & 2 & 1 \\
2 & -2 & -3 & 1 & 2 \\
-3 & -3 & 12 & -3 & -3 \\
2 & 1 & -3 & -2 & 2 \\
1 & 2 & -3 & 2 & -2
\end{array}\right) \\
&+\frac{\sin (2 \alpha)}{4 \sqrt{5}}\left(\begin{array}{ccccc}
0 & 0 & -1 & 0 & 1 \\
0 & 0 & 1 & -1 & 0 \\
-1 & 1 & 0 & 1 & -1 \\
0 & -1 & 1 & 0 & 0 \\
1 & 0 & -1 & 0 & 0
\end{array}\right)
\end{aligned}
$$

while $\mathbf{W}^{a_{g}}$ is trivially the unit matrix. We note that, even though the matrices $\mathbf{W}^{\tau}$, for different $\tau$ 's, do not commute, the eigenvector $(1,1,1,1,1) / \sqrt{5}$ is an eigenstate of each of them. Moreover, for each $\tau$ this eigenstate provides the largest eigenvalue

$$
\operatorname{Max}_{\text {eigenvalue }}\left(\mathbf{W}^{\tau}\right)=\sum_{n} W_{m n}^{\tau}=1
$$

(for any $m$ and $\tau$ ). This means that the totally-symmetric paired state, delocalized over all the five $H_{u}$ orbitals or bands, is favored by the couplings to all modes. It also implies that, contrary to molecular JT, the couplings to all modes cooperate evenly to this superconducting state, and contribute additively to $\lambda$. Totally equivalent (even if at first sight apparently different) results were derived for the $d=3$ case $\left(\mathrm{K}_{3} \mathrm{C}_{60}\right)$ in Refs. (Lannoo et al. 1991, Rice et al. 1991). We note, however, that the claim that orbital degeneracy enhances the superconducting $\lambda$ through a factor $d$ (Rice et al. 1991) is not really justified, as one must at the same time reduce the density of states from total to single-band, a factor $1 / d$ smaller. We also note that

$$
1=\sum_{n} W_{m n}^{\tau}=\frac{\sum_{m n} W_{m n}^{\tau}}{d}=\frac{\sum_{m n} V_{m n}^{\tau} V_{n m}^{\tau}}{d}=\frac{\sum_{m}\left[\left(\mathbf{V}^{\tau}\right)^{2}\right]_{m m}}{d}=\frac{\operatorname{Tr}\left[\left(\mathbf{V}^{\tau}\right)^{2}\right]}{d},
$$


which shows the identity of the $\tilde{\lambda}$ computed for superconductivity to the one obtained for transport in Eq. (20).

If, in the opposite limit, the pairs did not interfere between different bands, the effective $\lambda$ would be reduced by a factor $d$. Although we cannot identify a physical situation corresponding to this limit, we can assume that a general case will be intermediate between the limits (interfering/not-interfering pairs). For simplicity, we will stick here to the interfering limit.

In summary, is there any enhancement of superconductivity due to orbital degeneracy? We can still identify one possible source for that, namely Coulomb pseudopotential. In fact, we note that, although a large $\lambda$ due to tunneling of the Cooper pairs between different orbitals/bands, can be seen as orbital degeneracy enhancing the effective $\lambda$ to the highest eigenvalue of $\mathbf{W}$, there is no corresponding enhancement of the repulsive Coulomb pseudo-potential $\mu^{*}$, at least within the Migdal-Eliashberg theory. The reason is that the main contribution to the Coulomb pseudo-potential is a charge-charge repulsion which does not include tunneling processes between different bands, and being band-diagonal it does not get enhanced. In conclusion, in the above restricted sense, orbital degeneracy may in principle favor superconductivity.

\section{Discussion}

We have presented a density functional calculation of the linear coupling of holes/electrons in the $H_{u}$ and $T_{1 u}$ orbitals of the fullerene molecule to the intra-molecular vibrations. The coupling to holes is strongest for the $h_{g}$ modes, and among those to the lowest-frequency mode $h_{g}(1)$ around $270 \mathrm{~cm}^{-1}$. The linear static Jahn Teller distortion predicted for $\mathrm{C}_{60}^{+}$by these couplings corresponds to a $D_{5}$ distortion, with an energy gain of $71 \mathrm{meV}$.

The corresponding dynamical JT state expected with the calculated coupling parameters is a regular Berry-phase vibronic state of symmetry $H_{u}$, like the parent electronic state (Manini and De Los Rios 2000). There is no level crossing to a nondegenerate $A_{u}$ state, as would have hypothetically been possible on pure symmetry grounds, had the $D_{3}$ minima been the stable ones (Manini and De Los Rios 2000).

In order to connect with important solid state properties including transport and superconductivity we have formulated a theory of the Boltzmann relaxation time, and of the BCS-type pairing, suitable for an orbitally degenerate multiband case with Jahn Teller coupling. This confirms that the same parameter $\lambda$ determines both transport and superconducting properties of the multiband degenerate solid. As we previously observed, not all the computed $g_{i}$ (Table 2) are small parameters, and thus weak-coupling BCS theory is strictly not applicable for $\mathrm{C}_{60}^{n+}$. However, the overall $\lambda$ is still moderate. Our calculation neglects couplings to acoustic phonons and librations, which in principle should also contribute to e-ph scattering. In addition, similarly to 
Lannoo et al.'s calculation (Lannoo et al. 1991), we assume that the dispersion of the HOMO band and of the optical phonons has a negligible effect on the integrated value of $\lambda$. (This assumption was tested and proved correct in Ref. (Antropov et al. 1993) for the couplings to the LUMO band.) With all these approximations, Eq. (27) should provide a semi-quantitative estimate of the total e-ph scattering.

Assuming conservatively a total average density of states of $\sim 10 / 0.6 \mathrm{eV} \approx 17$ states $\mathrm{eV}^{-1}$ for the HOMO band (i.e. a single-band density of states $N_{1}(0)=1.7$ states $\mathrm{eV}^{-1}$ per band per spin), our calculated effective dimensionless $\lambda_{+}$for hole superconductivity in $\mathrm{C}_{60}$ is in conclusion about $\lambda_{+} \approx 0.47$. The Coulomb pseudopotential $\mu^{*}$ is not available yet, but possibly in the same range of values as for negative $\mathrm{C}_{60}$ $\left[\mu^{*} \sim 0.2 \div 0.3\right.$ (Gunnarsson et al. 1995, Gunnarsson and Zwicknagl 1992)]. With this value of $\lambda_{+}$, weak coupling would predict $T_{c} \sim 1.14 \hbar \omega_{\mathrm{D}} k_{\mathrm{B}}^{-1} \exp \left[-1 /\left(\lambda_{+}-\mu^{*}\right)\right] \sim 40 \mathrm{~K}$ for $\mu^{*}=0.2$, and $T_{c} \sim 5 \mathrm{~K}$ for $\mu^{*}=0.3$ (assuming a typical phonon energy $\omega_{D}$ of about $1500 \mathrm{~K}$ ). This seems of the correct order of magnitude, although somewhat on the low side, in comparison with $T_{c}=52 \mathrm{~K}$ found experimentally. However, it is difficult to justify weak coupling in this case.

The corresponding value $\lambda_{-}$which we obtain for electrons in the $T_{1 u}$ orbitals is, assuming the same bandwidth of $0.6 \mathrm{eV}$, thus again $N_{1}(0)=1.7$ states $\mathrm{eV}^{-1}$ per band per spin for the $T_{1 u}$ band, $\lambda_{-} \approx 0.33$. The factor $\lambda_{+} / \lambda_{-}=1.4$ of holes relative to electrons is in qualitative agreement with a larger $T_{c}$ of the former. Assuming the same typical phonon frequency, BCS would predict here $T_{c} \sim 0.8 \mathrm{~K}$ for $\mu^{*}=$ 0.2 , and $T_{c} \sim 0 \mathrm{~K}$ for $\mu^{*}=0.3$. That is obviously way smaller than the observed superconducting $T_{c}=10 \mathrm{~K}$ found experimentally in the field emission transistor (FET) experiment, let alone the higher values found in the fullerides.

Coming to transport, the measured resistivities for holes are larger than those of electrons, and this also agrees with a larger $\lambda$ value. Calculation of the $T$-linear high temperature resistivity

$$
\rho=\frac{\lambda_{t r} T}{4 \pi \omega_{p}^{2}}
$$

would however predict a moderately larger value for hole- than for electron-doped $\mathrm{C}_{60}$, at least assuming (somewhat arbitrarily) the same plasma frequencies for the same carrier densities. Quantitatively, Batlogg's FET data (Schön et al. 2000) differ strongly from this expectation. They, first of all, indicate a nonlinear temperature dependence, closer to $T^{2}$; secondly, they show values about 5 times larger for holes than for electrons. While there are second order processes (see Appendix) that would indeed yield a $T^{2}$ resistivity at low temperatures, we do not believe that they may explain the discrepancy here. Zettl and coworkers (Vareka and Zettl 1994) proposed that the apparent $T^{2}$ in the electron resistivity is an effect of thermal expansion, and showed that a linear $T$ increase is recovered at constant volume, for negative doping.

Recently Goldoni et al. measured by EELS the plasma frequency in $\mathrm{K}_{3} \mathrm{C}_{60}$. They found it slowly decreasing with temperature, its width growing approximately quadrat- 
ically with $T$. These data support the view that the $T^{2}$ resistivity is directly related with a $T^{-2}$ decrease of relaxation time, most likely linked with lattice expansion. It seems plausible that a similar physics could apply to holes too, in which case the predicted constant-pressure relaxation-time drop with temperature would also be non-linear, and quantitatively larger than the electron case. If, on the other hand, it became possible to obtain the constant-volume inverse relaxation time and resistivity, then, assuming the same plasma frequency $\omega_{p}$, its increase should be linear with $T$ with a slope 1.4 times larger than that of negatively-charged $\mathrm{C}_{60}$. This conjecture must await experimental test.

\section{Acknowledgements}

We are indebted to B. Batlogg, O. Gunnarsson and G. Santoro for useful discussions. This work was partly supported by the European Union, contract ERBFMRXCT970155 (TMR Fulprop), and by MURST COFIN99.

\section{Appendix: Quadratic Resistivity}

Within Fermi's golden rule, the vibron contribution to the electrical resistivity is exponentially decreasing if temperature is much smaller than the vibron frequencies. However, the above result is not true any more if higher order corrections are taken into account. Indeed, at second order, the electron-vibron coupling generates an effective electron-electron interaction. Since the electrons involved lie on a shell of width $T$ around the Fermi energy, the vibron-originated electron-electron interaction

$$
\begin{aligned}
V_{e l-e l}(\omega)= & -\sum_{\tau, i, m} \frac{g_{\tau i}^{2} \hbar \omega_{\tau i}}{4} \frac{\omega_{\tau i}^{2}}{\omega_{\tau i}^{2}-\omega^{2}} \\
& \frac{1}{V} \sum_{k p q} \sum_{\mu \nu \gamma \beta} \sum_{\sigma, \sigma^{\prime}} V_{\mu \nu}^{\tau(m)}(\alpha) V_{\gamma \beta}^{\tau(m)}(\alpha) d_{\mu, \sigma, k+q}^{\dagger} d_{\gamma, \sigma^{\prime}, p}^{\dagger} d_{\beta, \sigma^{\prime}, p+q} d_{\nu, \sigma, k},
\end{aligned}
$$

acts as if it were effectively unretarded, $\omega=0$. This interaction induces a $T^{2}$ inverse relaxation time at low temperatures. A rough estimation of the order of magnitude gives

$$
\begin{aligned}
\frac{1}{\tau} \propto & \frac{\left(k_{\mathrm{B}} T\right)^{2}}{5 \hbar} N_{1}\left(\epsilon_{F}\right)^{3} \sum_{i, j, \tau, \tau^{\prime}, m, m^{\prime}}\left(\frac{g_{\tau i}^{2} \hbar \omega_{\tau i}}{4}\right)\left(\frac{g_{\tau^{\prime} j}^{2} \hbar \omega_{\tau^{\prime} j}}{4}\right) \\
& \left\{\frac{5}{4}\left[\operatorname{Tr}\left(V^{\tau(m)} V^{\tau^{\prime}\left(m^{\prime}\right)}\right)\right]^{2}-\operatorname{Tr}\left(V^{\tau(m)} V^{\tau^{\prime}\left(m^{\prime}\right)} V^{\tau(m)} V^{\tau^{\prime}\left(m^{\prime}\right)}\right)\right\} .
\end{aligned}
$$




\section{References}

[1] Altmann, S. L., and Herzig, P., 1994, Point-Group Theory Table (Oxford Science Pub., Oxford).

[2] Antropov, V. P., Gunnarsson, O., and Liechtenstein, A. I., 1993, Phys. Rev. B 48, 7651 .

[3] Auerbach, A., Manini, N., and Tosatti, E., 1994, Phys. Rev. B 49, 12998.

[4] Baroni, S., Giannozzi, P., and Testa, A., 1987, Phys. Rev. Lett. 58, 1861; Dal Corso, A., Pasquarello, A., and Baldereschi, A., 1997, Phys. Rev. B 56, R11369; Baroni, S., de Gironcoli, S., Dal Corso, A., and Giannozzi, P., 2001, Rev. Mod. Phys. 73, in print.

[5] Brühwiler, P., Maxwell, A. J., Balzer, P., Andersson, S., Arvanitis, D., Karlsson, L., and Mårtensson, N., 1997, Chem. Phys. Lett. 279, 85.

[6] Butler, P. H., Point Group Symmetry Applications (Plenum, New York).

[7] Ceulemans, A., and Fowler, P. W., 1990, J. Chem. Phys. 93, 1221.

[8] Ceulemans, A., Fowler, P. W., and Vos, I., 1994, J. Chem. Phys. 100, 5491.

[9] Datars, W. R., and Ummat, P. K., 1995, Solid State Commun. 94, 649.

[10] De Los Rios, P., and Manini, N., 1997, in Recent Advances in the Chemistry, Physics of Fullerenes, Related Materials: Volume 5; in Recent Advances in the Chemistry and Physics of Fullerenes and Related Materials: Volume 5, edited by Kadish, K. M., and Ruoff, R. S. (The Electrochemical Society, Pennington), p. 468 .

[11] De Los Rios, P., Manini, N., and Tosatti, E., 1996, Phys. Rev. B 54, 7157.

[12] The C pseudopotential is described in Favot, F., and Dal Corso, A., 1999, Phys. Rev. B 60, 11427.

[13] Fowler, P. W., and Ceulemans, A., 1985, Mol. Phys. 54, 767.

[14] Giannozzi, P., and Baroni, S., 1994, J. Chem. Phys. 100, 8537.

[15] Goldoni, A., Sangaletti, L., Parmigiani, F., Comelli, G., and Paolucci, G., 2001, Phys. Rev. Lett., in print.

[16] Gunnarsson, O., Handschuh, H., Bechthold, P. S., Kessler, B., Ganteför, G., and Eberhardt, W., 1995, Phys. Rev. Lett. 74, 1875; Gunnarsson, O., 1995, Phys. Rev. B 51, 3493 (1995).

[17] Gunnarsson, O., and Zwicknagl, G., 1992, Phys. Rev. Lett. 69, 957-960. 
[18] Kohanoff, J., Andreoni, W., and Parrinello, M., 1992, Phys. Rev. B 46, 4371; Kohanoff, J., Andreoni, W., and Parrinello, M., 1992, Chem. Phys. Lett. 198, 472; Feuston, B. P., Andreoni, W., Parrinello, M., and Clementi, E., 1991, Phys. Rev. B 44, 4056.

[19] Lannoo, M., Baraff, G. A., and Schlüter, M., 1991, Phys. Rev. B 44, 12106.

[20] Manini, N., and De Los Rios, P., 1998, J. Phys.: Condens. Matter 10, 8485.

[21] Manini, N., and De Los Rios, P., 2000, Phys. Rev. B 62, 29.

[22] Manini, N., and Tosatti, E., 1998, Phys. Rev. B 58, 782.

[23] Manini, N., Tosatti, E., and Auerbach, A., 1994, Phys. Rev. B 49, 13008.

[24] Moate, C. P., Dunn, J. L., Bates, C. A., and Liu, Y. M., 1997, J. Phys.: Condens. Matter 9, 6049.

[25] Moate, C. P., O’Brien, M. C. M., Dunn, J. L., Bates, C. A., Liu, Y. M., and Polinger, V. Z., 1996, Phys. Rev. Lett. 77, 4362.

[26] Negri, F., Orlandi, G., and Zerbetto, F., 1988, Chem. Phys. Lett. 144, 31.

[27] Prassides, K., Dennis, T. J. S., Hare, J. P., Tomkinson, J., Kroto, H. W., Taylor, R., and Walton, D. R. M., 1991, Chem. Phys. Lett. 187, 455; Prassides, K., Christides, C., Rosseinsky, M. J., Tomkinson, J., Murphy, D. W., and Haddon, R. C., 1992, Europhys. Lett. 19, 629; Jishi, R. A., and Dresselhaus, M. S., 1992, Phys. Rev. B 45, 2597; Mitch, M. G., Chase, S. J., and Lannin, J. S., 1992, Phys. Rev. B 46, 3696.

[28] Rice, M. J., Choi, H. Y., and Wang, Y. R., 1991, Phys. Rev. B 44, 10414.

[29] Schön, J. H., Kloc, Ch., Haddon, R. C., and Batlogg, B., 2000, Science 288, 656; Schön, J. H., Kloc, Ch., and Batlogg, B., 2000, Nature 408, 549.

[30] Vanderbilt, D., 1990, Phys. Rev. B 41, 7892.

[31] Vareka, W. A., and Zettl, A., 1994, Phys. Rev. Lett. 72, 4121.

[32] Varma, C. M., Zaanen, J., and Raghavachari, K., 1991, Science 254, 989.

[33] Zhou, P., Wang, K. A., Wang, Y., Eklund, P. C., Dresselhaus, M. S., Dresselhaus, G., and Jishi, R. A., 1992, Phys. Rev. B 46, 2595. 